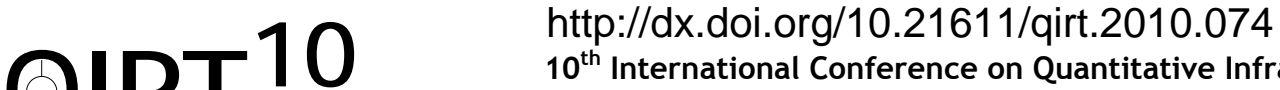 \\ $10^{\text {th }}$ International Conference on Quantitative InfraRed Thermography \\ July 27-30, 2010, Québec (Canada)
}

\section{Detecting the wastewater leakage from cesspool using noisy thermographic images}

\author{
by A. Lesniak* and B. Bukowska-Belniak* \\ *Geoinformatics \& Applied Computer Science Dept., AGH University of Science and Technology, Krakow, Poland, \\ lesniak@agh.edu.pl, bukowska@agh.edu.pl
}

\begin{abstract}
In following paper applicability of thermographic method for natural environment monitoring is discussed. Interpretation of low contrast thermographic images of a leaky cesspool is presented. The aims of the measurements were detection of wastewater leakage and precise determination of contaminated area. For noise reduction and thermal anomaly detection we propose the wavelet decomposition de-noising procedure combined with specially designed for such images segmentation process.
\end{abstract}

\section{Introduction}

Environmental monitoring is an important task, especially in areas exposed for pollution and surroundings contamination. Such kind of monitoring can be carry out in different scales and using different kind of measurements. It is focused on observations of atmosphere, ground and underground water, soil or noise level. Important kind of environmental monitoring is a wastewater leakage monitoring [1]. Physical measurements used in this case are based on measurements of different physical and chemical parameters. One of them is thermography. It allows to remote measurement of small and local temperatures of objects (e.a contaminated liquids or fluids with active endogenic chemical reactions). Thermografic monitoring of the leakages of contaminated substances is possible in cases when the temperature contrast exists. In many cases (e.g. in first example described below) contaminated water or savage in cesspools are warmer than the surroundings. However it is also possible that the contaminated water that leaks from a cesspool is colder than sun exposed ground as in our second example. Anyway if the temperature contrast exists it is possible to use thermographic method for remote detection and monitoring.

\section{Measurements}

For acquisition we have used thermographic camera VIGOcam v50, which works on microbolometric detector of size $384 \times 288$ pixels. The detector array with $35 \mu \mathrm{m}$ pixel and excellent optics, ensure enough for our aims thermal and spatial resolution. The atmospheric window of the camera is in the range $8-14 \mu \mathrm{m}$ of wavelength. It allows to precisely registering temperatures of objects even from long distance.

The VIGOcam v50 camera works in two ranges of temperature's measurements. In our experiment we have used the first one. It ranges from $-10^{\circ} \mathrm{C}$ to $150^{\circ} \mathrm{C}$. Thermal resolution of the camera in case of measurements, which are made when ambient temperature is equal to $30^{\circ} \mathrm{C}$ is equal to $0.08^{\circ} \mathrm{C}$.

\subsection{Leakage from cesspool.}

The aim of our experiments was inspection of temperature conditions in vicinity of leaky cesspools and possible location of wastewater leaks. In first experiment we acquired the images in early spring, at 1 o'clock at night. Maximum temperature of the air during the day was $18^{\circ} \mathrm{C}$, at night temperature decreased to $3^{\circ} \mathrm{C}$. Near the ground the temperature dropped under $0^{\circ} \mathrm{C}$ at night. The reason of such low ground temperature was frosty weather during the weeks before measurements. The near surface part of the ground was still frozen after winter and during the day only its surface was melted. The weather conditions during the measurements were good, there was no wind, only few clouds in the sky. There was no atmospheric precipitation during several days before measurements.

Thermographic measurements were done on the private possession where single house was built. The building is connected to household sewage treatment plant. In time of measurements sewage system was switched to unused cesspool and the cesspool was felt up with refuse water. A few thermal images in cesspool surroundings from different sides were registered. Repeated measurements minimize error of temperature acquiring and verify repeatability of measurements. An example of registered image is presented in figure 1. In this image the area with high temperature in neighbourhood of cesspool's cover can be observed. Temperature in the presented image varies from $-3.9^{\circ} \mathrm{C}$ to $2^{\circ} \mathrm{C}$ and thermal resolution is 
about $0.1^{\circ} \mathrm{C}$, so the contrast isn't very good. Cause of the high temperatures in neighbourhood of cesspool can be leaky cesspool chamber and leaks of wastewater, which have higher temperature than in distant places.

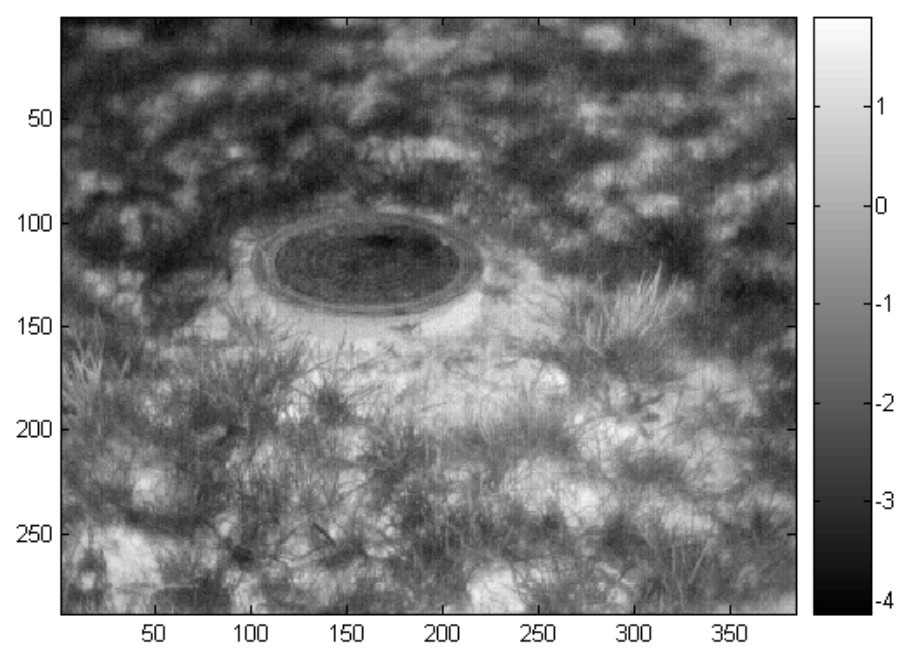

Fig. 1. Thermographic image of small cesspool and possible leakage of contaminated water. Color bar in Celsius degree.

\subsection{Illegal leakage of wastewater to the river.}

Second experiment was an observation of illegal wastewater discharge to the river. The measurements took place in March, around noon. Weather was sunny and windless. Temperature of the air was about $14^{\circ} \mathrm{C}$. Leakage was localized in south part of Krakow (Poland). In neighbourhood of the leakage there are cesspools of single-family houses without sewerage system. The reason of the leakage was an untight cesspools or conscious actions of inhabitants. Such leakages have a serious impact on its surroundings and can be reason of environment degradation. It is very hard to detect and point the source of the contamination and persons responsible for this kind of offence. Figure 2 presents location scheme of leakage surroundings.

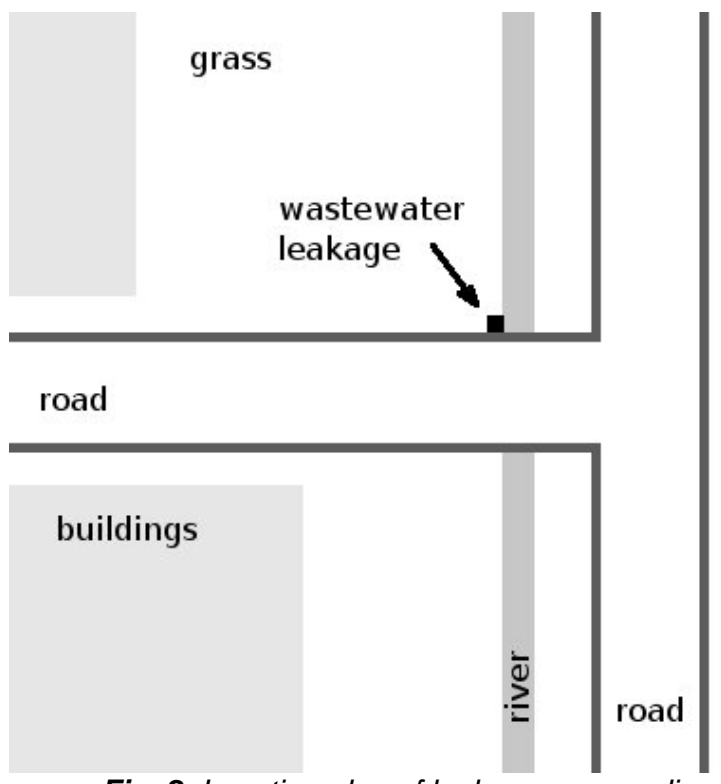

Fig. 2. Location plan of leakage surroundings 
The leakage was localized under the bridge on the slope of the riverbank, about $1 \mathrm{~m}$ above the water level. The wastewater flowed from the pipe, but it was impossible to find from which possession this pipe starts. In figure $3 a$ thermal image is presented. Minimal value of temperature in this picture is equal $4.9^{\circ} \mathrm{C}$, maximum value is equal $13.94^{\circ} \mathrm{C}$. Temperature of the leakage (about $5.5^{\circ} \mathrm{C}$ ) is lower than temperature of water in the river (about $8^{\circ} \mathrm{C}$ ). The reason of the temperature contrast of the wastewater and river water could be the low temperature of the ground, the significant pipe length and low intensity of the flow. Figure $3 b$ presents an image made in visible light of the same area.
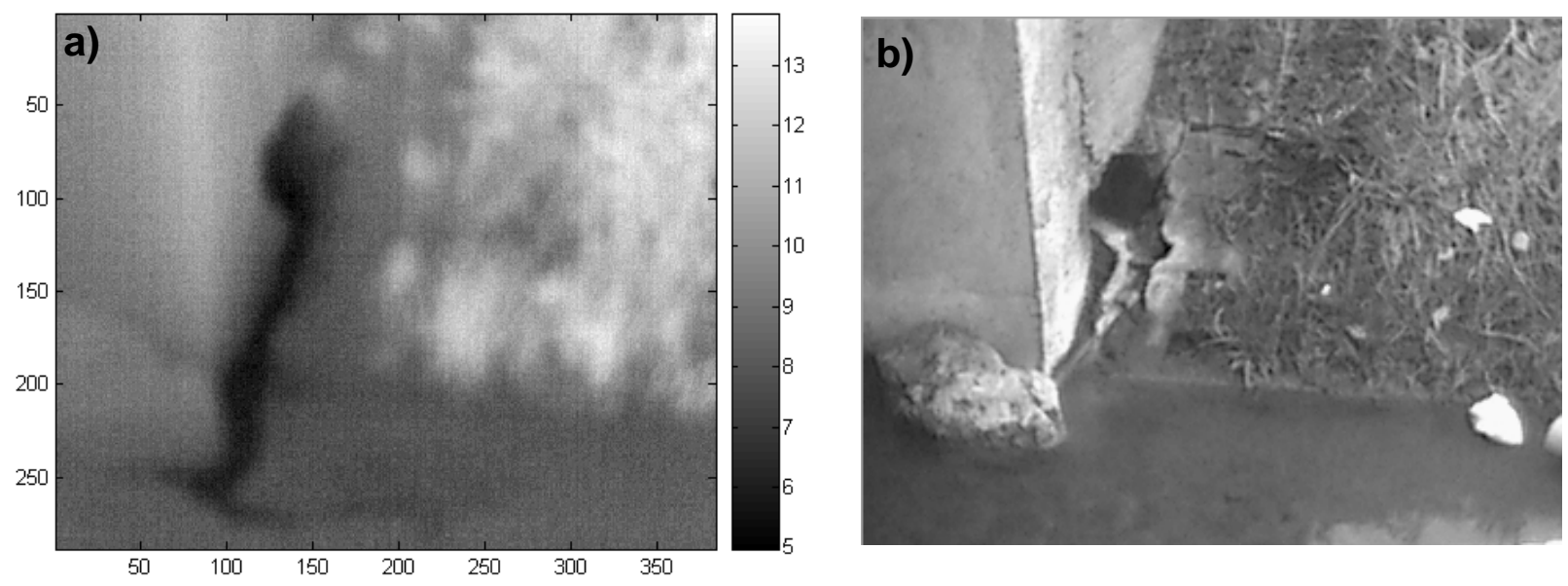

Fig. 3. Thermographic image of wastewater leakage (a) and image in visible light of the same area (b). Color bar in Celsius degree.

\section{Processing method}

The aim of the processing of registered images is rejection of the random noise and segmentation of the images. The perfect result is achieved when the image is decomposed into two parts - the first one that includes region with anomalous temperature and the second - the background. The precise evaluation of the anomalous temperature area can be important in detailed analysis of the direction of polluted water flows and the scale of the contamination. The essential feature of the thermographic images gathered outdoor in unstable temperature conditions is high noise level and fuzzy picture of regions of interests. The noise has a different scale which varies from pixels to tens of pixels. The shape of the noisy elements also varies. They can be elongated or round-shaped.

To unify the procedure of noise reduction we have used the multiscale analysis. Two dimensional (2D) multiscale analysis is based on 1D wavelet analysis of signals. The discrete version of wavelet transform (DWT) projects signal $f(x)$ onto two spaces: the first one called approximation space $\Phi$ and detail space $\Psi$. Signal decomposition is achieved by discrete filtering procedure applied to downsampling signal. As a result we obtain the signal decomposed into so called scale function $\varphi(x)$ and wavelet function $\psi(x)$ e.a [2]:

$$
f(x)=\frac{1}{\sqrt{M}} \sum_{k} W_{\varphi}\left(j_{0}, k\right) \varphi_{j_{0}, k}(x)+\sum_{j=j_{0}}^{J-1} \sum_{k} W_{\psi}(j, k) \psi_{j, k}(x)
$$

where

$$
W_{\varphi}\left(j_{0}, k\right)=\frac{1}{\sqrt{M}} \sum_{x} f(x) \varphi_{j_{0}, k}(x) \quad W_{\psi}(j, k)=\frac{1}{\sqrt{M}} \sum_{x} f(x) \psi_{j, k}(x)
$$

and usually $j_{0}=0 \quad M=2^{J} \quad j=0,1,2, \ldots, J-1 \quad k=0,1,2, \ldots, 2^{j}-1$

Scale and wavelet functions obey the refinement equations:

$$
\varphi(x)=\sum_{n} h_{\varphi}(n) \sqrt{2} \varphi(2 x-n)
$$




$$
\psi(x)=\sum_{n} h_{\psi}(n) \sqrt{2} \varphi(2 x-n)
$$

where $h_{\varphi}(n)$ are the coefficients of the discrete lowpass filter and $h_{\psi}(n)$ are coefficients of highpass filter.

The images (e.a 2D signals) are decomposed onto four subspaces: the scaling functions $\varphi(x, y)=\varphi(x) \varphi(y)$, and the wavelet functions: the vertical $\psi^{V}(x, y)=\varphi(x) \psi(y)$, the horizontal $\psi^{H}(x, y)=\varphi(x) \psi(y)$ and the diagonal one $\psi^{D}(x, y)=\psi(x) \psi(y)$. The formula for the image decomposition in 2D case is similar to the 1D:

$$
f(x, y)=\frac{1}{\sqrt{M N}} \sum_{m=0}^{M-1} \sum_{n=0}^{N-1} W_{\varphi}\left(j_{0}, m, n\right) \varphi_{j_{0}, m, n}(x, y)+\frac{1}{\sqrt{M N}} \sum_{i=H, V, D} \sum_{j=j_{0}}^{J-1} \sum_{m=0}^{M-1} \sum_{n=0}^{N-1} W_{\psi}^{i}\left(j_{0}, m, n\right) \psi_{j_{0}, m, n}^{i}(x, y)
$$

The values of the coefficients $W_{\psi}^{i}$ depends on the size of the objects in the image.

To detect the leakage and determine the contaminated area we apply two steps procedure. The first step is wavelet de-noising procedure. We have used symmetrical wavelets approximation to remove the details (the tufts of grass visible in figure 4) from the image. They can be treated as a noise of coarse nature. To achieve it we synthesised image only from coefficients $W_{\psi}^{i}$ related to high amplitudes wavelets of each level of decomposition. The results can be seen in figure $4 a$. In the second step we synthesized image using only coefficients of the scale function at J-1 level of decomposition. In the first example we have used fifth level. It allows us to visualize the area of relative high temperature. In Figure $4 \mathrm{~b}$ we can see the leakage area estimated from thermographic image. Its boundary is isothermal line that surrounds the area of the high temperature that fulfil the condition of the smallest spatial variance. I means that other isotherm surrounded area has a larger variance than the chosen one.

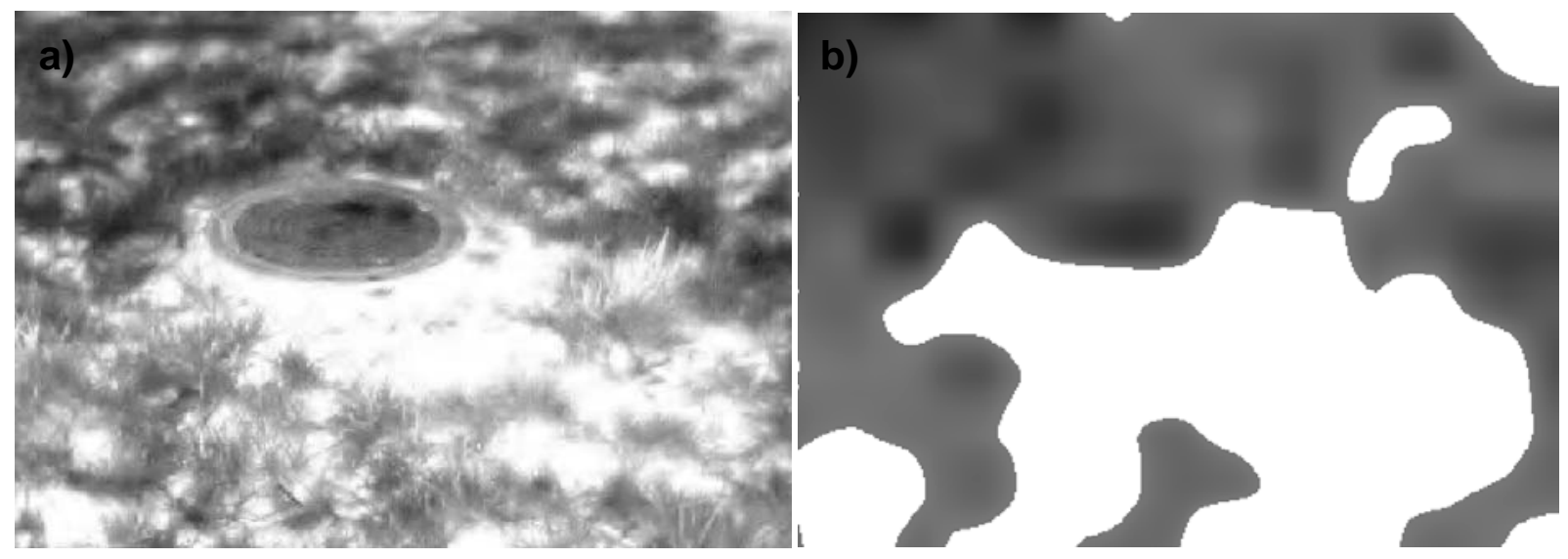

Fig. 4. Image of cesspool with reduced noise (a) and contaminated area around cesspool reconstructed from thermographic image (b)

The analogous procedure was applied to the image of the wastewater leakage. The noise that contaminate the image is now more fine. The black area covers the low temperature leakage from the pipe. It allows to estimate the area of the leakage on the slope of the river bank as well as the area of water mixing in the riverbed. In this case the level used for decomposition was equal to four. 

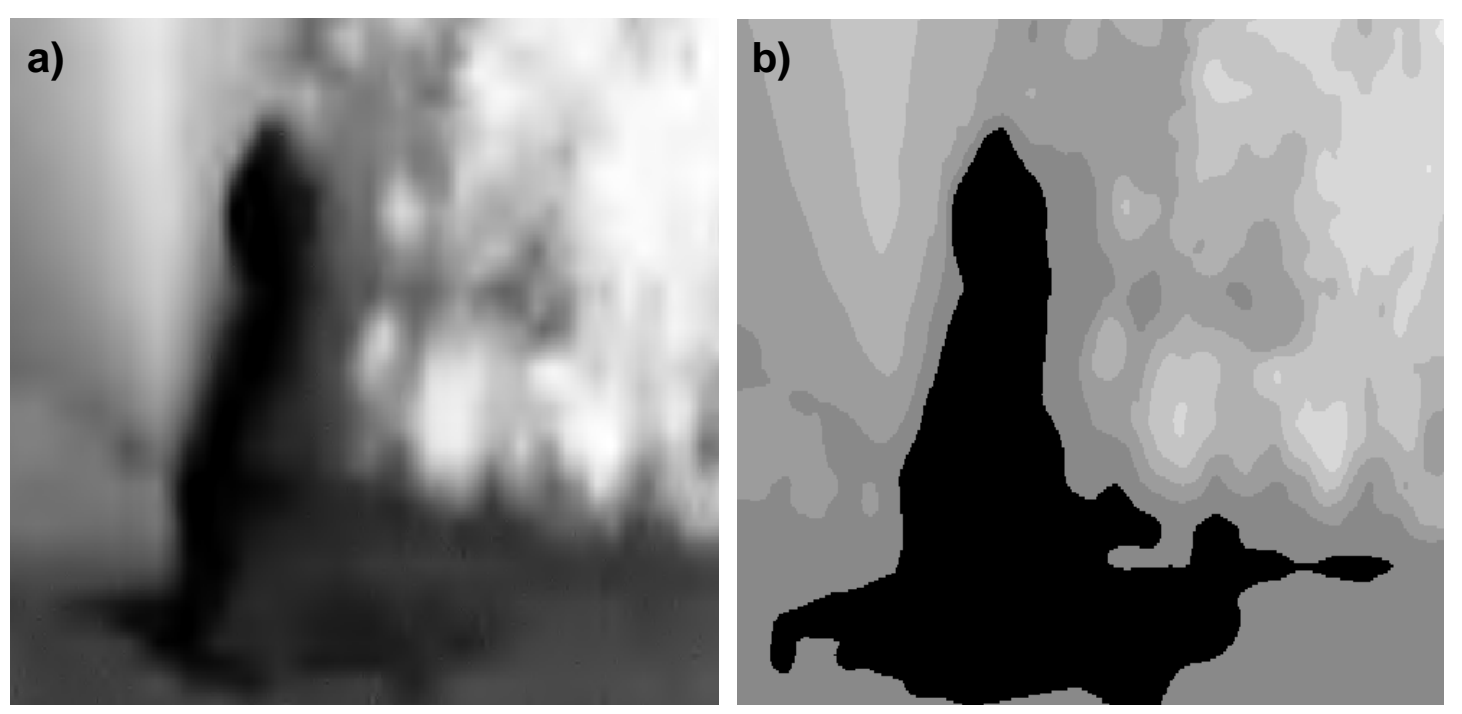

Fig. 4. Image of the wastewater leakage with reduced noise (a) and area contaminated by leakage reconstructed from thermographic image (b)

\section{Results and conclusions}

Using the described method we were in a position to determine the area contaminated by water that leaked from overflowing cesspool. The proposed processing procedure works well in spite of the sign of temperature anomaly and level of the noise. To perform the analysis we have to decide about few parameters. The first is the specific wavelet family $\psi(x)$ has to be chosen. We are limited to orthogonal wavelets and numerous experiments convinced us that so called symmetrical wavelets are the most suited ones for our purposes. We have to decided also about the level of decomposition. For our filtering purposes this level is related to the nature of the noise, exactly to the autocorrelation coefficient of the noise. For larger coefficient the level of decomposition has to be larger too.

The special processing method proposed above allows us to determining the area of the leakage in spite of grass and low temperature difference between clear and polluted soil. The method of measurements and processing is best suited for automatic detection and warning. Of course the thermography can only be auxiliary technique of sewage migration monitoring.

\section{Acknowledgments}

This paper was written as part of the statutory research project of the Department of Geoinformatics and Applied Computer Science, Faculty of Geology, Geophysics and Environmental Protection, AGH University of Science and Technology.

\section{REFERENCES}

[1] Bukowska-Belniak B., Leśniak A., "Application of Thermographic Research in Environmental Protection", Polish Journal of Environmental Studies, Olsztyn (Poland), Vol. 18, No. 3A, p. 38-43, 2009.

[2] Gonzalez, R.C., Woods, R.E., Digital Image Processing, Prentice Hall Inc., 2002 